\title{
The Feichtinger Conjecture for Wavelet Frames, Gabor Frames and Frames of Translates
}

\author{
Marcin Bownik and Darrin Speegle
}

Abstract. The Feichtinger conjecture is considered for three special families of frames. It is shown that if a wavelet frame satisfies a certain weak regularity condition, then it can be written as the finite union of Riesz basic sequences each of which is a wavelet system. Moreover, the above is not true for general wavelet frames. It is also shown that a sup-adjoint Gabor frame can be written as the finite union of Riesz basic sequences. Finally, we show how existing techniques can be applied to determine whether frames of translates can be written as the finite union of Riesz basic sequences. We end by giving an example of a frame of translates such that any Riesz basic subsequence must consist of highly irregular translates.

\section{Introduction and Statements of Main Results}

A frame is a collection of elements $\left\{e_{i}: i \in I\right\}$ in a Hilbert space $\mathcal{H}$ such that there exist positive constants $A$ and $B$ such that for every $h \in \mathcal{H}$,

$$
A\|h\|^{2} \leq \sum_{i \in I}\left|\left\langle h, e_{i}\right\rangle\right|^{2} \leq B\|h\|^{2} .
$$

A frame $\left\{e_{i}: i \in I\right\}$ is bounded if

$$
\inf _{i \in I}\left\|e_{i}\right\|>0
$$

(Note that it is automatic that $\sup _{i \in I}\left\|e_{i}\right\|<\infty$.) A sequence $\left\{e_{i}: i \in I\right\}$ is said to be a Riesz basic sequence if it is a Riesz basis for its closed linear span, i.e., there exist $K_{1}, K_{2}>0$ such that for every family of scalars $\left\{a_{i}: i \in I\right\}$

$$
K_{1} \sum_{i \in I}\left|a_{i}\right|^{2} \leq\left\|\sum_{i \in I} a_{i} e_{i}\right\|^{2} \leq K_{2} \sum_{i \in I}\left|a_{i}\right|^{2} .
$$

Note that for Riesz basic sequences we always have

$$
0<\inf _{i \in I}\left\|e_{i}\right\| \leq \sup _{i \in I}\left\|e_{i}\right\|<\infty .
$$

With this notation, one can state the Feichtinger conjecture:

Received by the editors July 5, 2004; revised January 21, 2005.

The first author was partially supported by the NSF grant DMS-0441817.

AMS subject classification: 42B25, 42B35, 42C40.

Keywords: frame, Riesz basic sequence, wavelet, Gabor system, frame of translates, paving conjecture. (C) Canadian Mathematical Society 2006. 
Conjecture 1.1 (Feichtinger) Every bounded frame can be written as the finite union of Riesz basic sequences.

The Feichtinger conjecture has received a fair amount of recent interest $[6,7,13]$, and it has been shown to be related to several famous open problems in analysis. In particular, in [6], it is shown that the conjectured generalization of the BourgainTzafriri restricted invertibility theorem is equivalent to the Feichtinger conjecture. It is also shown that the paving conjecture of Bourgain-Tzafriri (which is known to be equivalent to the Kadison-Singer conjecture) implies the Feichtinger conjecture. For precise statements of these conjectures and references, see [6].

In this paper, we consider the Feichtinger conjecture for special systems of frames such as wavelet and Gabor frames and frames of translates. In particular, we are investigating a stronger version of the Feichtinger conjecture, which asks whether a bounded frame of a certain type (such as wavelet, Gabor, or frame of translates) can be decomposed as the finite union of Riesz sequences of the same type.

The answer to this question depends on the type of frame at hand. Namely, we prove that wavelet frames satisfying certain weak decay and regularity conditions satisfy the stronger Feichtinger property, whereas wavelet frames without this additional regularity condition in general do not satisfy this property. In contrast, we show that a significant class of Gabor systems satisfying a certain compatibility condition on their generating lattices always have this property regardless of any regularity conditions. Finally, for frames of translates the situation with regard to the stronger Feichtinger property is the same as for wavelets. Nevertheless, we show that the Feichtinger conjecture leads to some interesting number theoretic problems involving the quantitative versions of van der Waerden's theorem.

The plan of this paper is as follows. In Section 2, we introduce the notion of localization for wavelet frames associated with general expansive dilations and we show that for such frames the stronger Feichtinger conjecture holds. We prove that this result is optimal in the sense that it does not hold for general wavelet frames without a localization property. In Section 3, we show that every sup-adjoint Gabor frame can be written as the finite union of Riesz basic sequences, generalizing results of Ron and Shen [21] and Casazza et al. [6].

In Section 4, we study frames of subspaces of $L^{2}(\mathbb{R})$ of the form $\mathcal{S}_{f}=\{f(x+k)$ : $k \in \Gamma\}$. We show that if $f(x)$ has good decay and $\mathcal{S}_{f}$ is a frame of a subspace, then $\mathcal{S}_{f}$ can be written as the finite union of Riesz basic sequences. Then we consider the following:

Question 1.2 Suppose $\hat{f}=\chi_{U}$, where $U \subset \mathbb{R}$, and $\mathcal{S}_{f}=\{f(x+k): k \in \mathbb{Z}\}$ is a frame for its closed linear span. Can $\mathcal{S}_{f}$ be written as a finite union of Riesz basic sequences?

Most interesting in this section is an example of a function $f(x)$ of the type $\hat{f}=$ $\chi_{U}$ such that $\mathcal{S}_{f}$ is a frame of a subspace, but such that if $N \subset \mathbb{Z}$ has arbitrarily long arithmetic progressions of a certain type, then $\{f(x+k): k \in N\}$ is not a Riesz basic sequence. In particular, a random subset of $\mathcal{S}_{f}$ almost surely is not a Riesz basic sequence; see Theorem 4.16 for details. This example is also compared 
to quantitative versions of van der Waerden's theorem to conclude the section. Note that there is a close connection between the Feichtinger conjecture in this setting and the paving conjecture for Laurent operators. This connection is also briefly explained in Section 4.

\subsection{Some Background Tools}

We start by recalling basic definitions and properties of non-isotropic Euclidean spaces associated with general expansive dilations. A real $n \times n$ matrix $A$ is an $e x-$ pansive matrix, often called a dilation, if $\min _{\lambda \in \sigma(A)}|\lambda|>1$, where $\sigma(A)$ is the set of all eigenvalues (the spectrum) of $A$. A fundamental notion in our study is a quasinorm $\rho_{A}$ associated with $A$.

Definition 1.3 A quasi-norm associated with an expansive matrix $A$ is a measurable mapping $\rho_{A}: \mathbb{R}^{n} \rightarrow[0, \infty)$ satisfying

$$
\begin{array}{clrl}
\rho_{A}(x) & >0 & & \text { for } x \neq 0, \\
\rho_{A}(A x) & =|\operatorname{det} A| \rho_{A}(x) & & \text { for } x \in \mathbb{R}^{n}, \\
\rho_{A}(x+y) & \leq H\left(\rho_{A}(x)+\rho_{A}(y)\right) & & \text { for } x, y \in \mathbb{R}^{n},
\end{array}
$$

where $H \geq 1$ is a constant.

Here, we will only list a few basic properties of quasi-norms $\rho_{A}$, which will be used subsequently. For more details we refer to $[3,18]$. We recall that all quasinorms associated to a fixed dilation $A$ are equivalent, see [3, Lemma 2.4]. Moreover, there always exist a quasi-norm $\rho_{A}$, which is $C^{\infty}$ on $\mathbb{R}^{n}$ except the origin, see [18].

Proposition 1.4 For any expansive matrix $A$ and $\epsilon>0$,

$$
\int_{B(0,1)} \rho_{A}(x)^{\epsilon-1} d x<\infty \text { and } \int_{\mathbb{R}^{n} \backslash B(0,1)} \rho_{A}(x)^{-1-\epsilon} d x<\infty
$$

Proposition 1.4 follows from

$$
(1 / c) r \leq\left|\left\{x \in \mathbb{R}^{n}: \rho_{A}(x)<r\right\}\right| \leq c r \quad \text { for all } r>0 .
$$

Lemma 1.5 Suppose $A$ is an expansive matrix, and $\lambda_{-}$and $\lambda_{+}$are any positive real numbers such that $1<\lambda_{-}<\min _{\lambda \in \sigma(A)}|\lambda|$ and $\lambda_{+}>\max _{\lambda \in \sigma(A)}|\lambda|$. Let

$$
\zeta_{-}:=\frac{\ln \lambda_{-}}{\ln |\operatorname{det} A|}, \quad \zeta_{+}:=\frac{\ln \lambda_{+}}{\ln |\operatorname{det} A|} .
$$

Then for any quasi-norm $\rho_{A}$ there exists a constant $C$ such that,

$$
C^{-1} \rho_{A}(x)^{\zeta_{-}} \leq|x| \leq C \rho_{A}(x)^{\zeta_{+}} \quad \text { if } \rho_{A}(x) \geq 1
$$


and

$$
C^{-1} \rho_{A}(x)^{\zeta_{+}} \leq|x| \leq C \rho_{A}(x)^{\zeta_{-}} \quad \text { if } \rho_{A}(x) \leq 1
$$

Furthermore, if $A$ is diagonalizable over $\mathbb{C}$, then we may take $\lambda_{-}=\min _{\lambda \in \sigma(A)}|\lambda|$ and $\lambda_{+}=\max _{\lambda \in \sigma(A)}|\lambda|$.

For any $j \in \mathbb{Z}$ and $k \in \mathbb{Z}^{n}$, let $Q_{j, k}=A^{-j}\left([0,1]^{n}+k\right)$ be the dilated cube, and $x_{Q_{j, k}}=A^{-j} k$ be its "lower-left corner". Let

$$
\mathcal{Q}=\left\{Q_{j, k}: j \in \mathbb{Z}, k \in \mathbb{Z}^{n}\right\}
$$

be the collection of all dilated cubes. Given a function $\psi$ on $\mathbb{R}^{n}$ we define

$$
\psi_{Q}(x)=|\operatorname{det} A|^{j / 2} \psi\left(A^{j} x-k\right)=|Q|^{-1 / 2} \psi\left(A^{j}\left(x-x_{Q}\right)\right) \quad \text { for } Q=Q_{j, k} \in Q
$$

Equivalently, if $Q=A^{-j}\left([0,1]^{n}+k\right) \in Q$, then $\psi_{Q}=D^{j} T_{k} \psi$, where $D$ is a dilation operator $D f(x)=|\operatorname{det} A|^{1 / 2} f(A x)$ and $T_{k}$ is a translation operator $T_{k} f(x)=$ $f(x-k)$.

\section{Affine Localized Frames}

Gröchenig [13] introduced a notion of localized frames and showed that the Feichtinger conjecture holds for this class of frames. While his definition works very well for systems generated by families of unitary operators with compatible actions on $L^{2}\left(\mathbb{R}^{n}\right)$, such as translations and modulations in the case of Gabor systems, there is a natural need to have a better adapted notion of localization for systems which are generated by families of operators with less compatible actions. The most prominent example of such system is an affine system generated by translates and dilates of a frame wavelet.

While it seems that there is no single universal notion of frame localization working for all imaginable frames in $L^{2}\left(\mathbb{R}^{n}\right)$, we propose that the following definition gives a satisfactory answer in the case of wavelet systems. Our definition is motivated by the almost diagonal condition of Frazier and Jawerth $[9,10,11]$ from the study of isotropic Besov and Triebel-Lizorkin spaces and their anisotropic analogues for expansive dilations introduced by the first author and Ho [4].

Definition 2.1 We say that a collection $\left\{\Psi_{Q}: Q \in Q\right\}$ is affine localized if there exists $C, \theta>0$ such that

$$
\left|\left\langle\Psi_{Q}, \Psi_{P}\right\rangle\right| \leq C \kappa_{Q P}(\theta) \quad \text { for all } Q, P \in \mathcal{Q},
$$

where

$$
\kappa_{Q P}(\theta)=\left(1+\frac{\rho_{A}\left(x_{Q}-x_{P}\right)}{\max (|P|,|Q|)}\right)^{-1-\theta} \min \left[\left(\frac{|Q|}{|P|}\right)^{1 / 2+\theta},\left(\frac{|P|}{|Q|}\right)^{1 / 2+\theta}\right] .
$$


Remark 2.2 We remark that $\Psi_{Q}$ should only be understood as a function indexed by $Q \in Q$, which is not necessarily given by (1.5).

Definition 2.3 We say that $\Psi_{Q}(x)$ is a molecule supported near $Q \in \mathcal{Q}$ with $|Q|=$ $|\operatorname{det} A|^{-j}$ and $j \in \mathbb{Z}$, if there exist $M>1$ and $\delta>0$ such that

$$
\begin{gathered}
\left|\Psi_{Q}(x)-\Psi_{Q}(y)\right| \leq \frac{|\operatorname{det} A|^{j(1 / 2+\delta)} \rho_{A}(x-y)^{\delta}}{\left(1+\rho_{A}\left(A^{j}\left(x-x_{Q}\right)\right)\right)^{M}} \\
\quad \text { for all } x, y \in \mathbb{R}^{n}, \rho_{A}(x-y) \leq|\operatorname{det} A|^{-j}, \\
\left|\Psi_{Q}(x)\right| \leq \frac{|\operatorname{det} A|^{j / 2}}{\left(1+\rho_{A}\left(A^{j}\left(x-x_{Q}\right)\right)\right)^{M}} \quad \text { for all } x \in \mathbb{R}^{n}, \\
\int_{\mathbb{R}^{n}} \Psi_{Q}(x) d x=0 .
\end{gathered}
$$

We say that a collection $\left\{\Psi_{Q}\right\}_{Q \in Q}$ is a family of molecules, if each $\Psi_{Q}$ is a molecule supported near $Q$ with the same $M>1$ and $\delta>0$.

Therefore, a molecule $\Psi_{Q}$ must satisfy certain smoothness (2.2) and decay (2.3) conditions appropriately localized to a dilated cube $Q$ in addition to the usual vanishing moment condition (2.4). We also mention that the smoothness condition (2.2) in Definition 2.3 can be replaced by

$$
\begin{aligned}
& \left|\Psi_{Q}(x)-\Psi_{Q}(y)\right| \\
& \quad \leq|\operatorname{det} A|^{j(1 / 2+\delta)} \rho_{A}(x-y)^{\delta} \sup _{\rho_{A}(z) \leq \rho_{A}(x-y)}\left(1+\rho_{A}\left(A^{j}\left(x-z-x_{Q}\right)\right)\right)^{-M}
\end{aligned}
$$

to obtain an equivalent definition of a molecule supported near $Q$ modulo a multiplicative constant independent of $Q$. A condition of the type (2.5) is more commonly used, and it was introduced by Frazier and Jawerth for the dyadic dilation $A=2$ Id in [10, Section 3]. To see the equivalence of (2.2) and (2.5) it suffices to make two observations. First, we have

$$
\begin{aligned}
1+\rho_{A}\left(A^{j}\left(x-x_{Q}\right)\right) & \leq \sup _{\rho_{A}(z) \leq \rho_{A}(x-y)} 1+\rho_{A}\left(A^{j}\left(x-z-x_{Q}\right)\right) \\
& \leq(H+1)\left(1+\rho_{A}\left(A^{j}\left(x-x_{Q}\right)\right)\right) \quad \text { for } \rho_{A}(x-y) \leq|\operatorname{det} A|^{-j}
\end{aligned}
$$

Second, if $\rho_{A}(x-y)>|\operatorname{det} A|^{-j}$, then (2.3) immediately implies (2.5), with the right-hand side having an additional factor of 2 .

Frequently, we will be interested in families of molecules generated by translates and dilates of a single function $\psi$. In this case, the following remark is relevant. 
Remark 2.4 Suppose that a function $\psi(x)$ satisfies

$$
\begin{gathered}
|\psi(x)-\psi(y)| \leq \frac{\rho_{A}(x-y)^{\delta}}{\left(1+\rho_{A}(x)\right)^{M}} \quad \text { for } \rho_{A}(x-y) \leq 1 \\
|\psi(x)| \leq\left(1+\rho_{A}(x)\right)^{-M} \\
\int_{\mathbb{R}^{n}} \psi(x) d x=0
\end{gathered}
$$

for some $\delta>0$ and $M>1$. Then the affine system $\left\{\psi_{Q}: Q \in \mathcal{Q}\right\}$ given by (1.5) forms a family of molecules. Easy verification of this fact is left to the reader.

Also, for those who do not like the use of the quasi-norm $\rho_{A}$, one can write a slightly stronger version of conditions (2.6) and (2.7), where $\rho_{A}$ is replaced by the standard Euclidean distance $|\cdot|$. Indeed, by Lemma 1.5 it suffices to assume that $\psi(x)$ satisfies $(2.8)$ and

$$
\begin{gathered}
|\psi(x)-\psi(y)| \leq \frac{|x-y|^{\delta}}{(1+|x|)^{M / \zeta_{-}}} \text {for }|x-y| \leq 1 \\
|\psi(x)| \leq(1+|x|)^{-M / \zeta_{-}}
\end{gathered}
$$

for some $\delta>0$ and $M>1$, in order to conclude that for sufficiently small constant $c>0,\left\{c \psi_{Q}: Q \in \mathcal{Q}\right\}$ given by (1.5) is a family of molecules in the sense of Definition 2.3. Here, $\zeta_{-}$is the same as in Lemma 1.5. In particular, for the dyadic dilation $A=2$ Id in $\mathbb{R}^{n}$, we have $\zeta_{-}=\zeta_{+}=1 / n$.

We are now ready to state the key result showing that families of molecules must satisfy almost diagonal estimates.

Lemma 2.5 Suppose $\left\{\Phi_{Q}\right\}_{Q}$ and $\left\{\Psi_{Q}\right\}_{Q}$ are families of molecules. Then there exist $C>0$ and $\theta>0$, such that

$$
\left|\left\langle\Psi_{P}, \Phi_{Q}\right\rangle\right| \leq C \kappa_{Q P}(\theta) \quad \text { for all } Q, P \in \mathcal{Q} \text {. }
$$

In particular, any family of molecules $\left\{\Psi_{Q}\right\}_{Q}$ is affine localized.

Lemma 2.5 was originally proved in the usual dyadic case by Frazier and Jawerth [10], in a much greater generality than needed here, for molecules with possible higher degrees of smoothness. In the case of general expansive dilations, the same was shown in [4]. Since the notion of smooth molecules in [4] is more restrictive than our Definition 2.3, we will give a proof of Lemma 2.5. We will need the following two elementary facts.

Lemma 2.6 Suppose that $j \in \mathbb{Z}, j \geq 0, \theta>0$, and $x \in \mathbb{R}^{n}$. Then there exists a constant $C>0$, which is independent of $j$ and $x$, such that

$$
\sum_{k \in \mathbb{Z}^{n}}\left(1+\rho_{A}\left(A^{-j} k+x\right)\right)^{-1-\theta} \leq C|\operatorname{det} A|^{j} .
$$


Proof First, note that Proposition 1.4 and a change of variables yield

$$
\int_{\mathbb{R}^{n}}\left(1+\rho_{A}\left(A^{-j} z+x\right)\right)^{-1-\theta} d z \leq C|\operatorname{det} A|^{j} .
$$

Next, it suffices to split the domain of integration into cubes $\mathbb{R}^{n}=\bigcup_{k \in \mathbb{Z}^{n}}\left(k+[0,1]^{n}\right)$ and use the fact that for $z \in k+[0,1]^{n}$,

$$
\rho_{A}\left(A^{-j} z+x\right) \leq H\left(\rho_{A}\left(A^{-j} k+x\right)+\sup _{y \in[0,1]^{n}} \rho_{A}\left(A^{-j} y\right)\right) \leq \tilde{C}\left(1+\rho_{A}\left(A^{-j} k+x\right)\right)
$$

Lemma 2.7 Suppose that for some $\theta>0, R>1+\theta$, and $i, j \in \mathbb{Z}, i \geq j$, and $x_{0} \in \mathbb{R}^{n}$, we have

$$
\begin{gathered}
|g(x)| \leq|\operatorname{det} A|^{j / 2}\left(1+\rho_{A}\left(A^{j} x\right)\right)^{-R}, \\
|g(x)-g(y)| \leq \frac{|\operatorname{det} A|^{j(1 / 2+\theta)} \rho_{A}(x-y)^{\theta}}{\left(1+\rho_{A}\left(A^{j} x\right)\right)^{R}} \text { for } \rho_{A}(x-y) \leq|\operatorname{det} A|^{-j}, \\
|h(x)| \leq|\operatorname{det} A|^{i / 2}\left(1+\rho_{A}\left(A^{i}\left(x-x_{0}\right)\right)\right)^{-R}, \\
\int_{\mathbb{R}^{n}} h(x) d x=0 .
\end{gathered}
$$

Then there exists a constant $C>0$ depending only on $\theta$ and $R$, such that

$$
|(g * h)(x)| \leq C|\operatorname{det} A|^{-(i-j)(1 / 2+\theta)}\left(1+\rho_{A}\left(A^{j}\left(x-x_{0}\right)\right)\right)^{-R} .
$$

Proof Using translation and dilation, we may assume that $j=0$ and $x_{0}=0$. Indeed, if $g$ and $h$ satisfy (2.12)-(2.14) for some $i_{0} \geq j_{0} \in \mathbb{Z}$ and $\tilde{x}_{0} \in \mathbb{R}^{n}$, then $D^{-j_{0}} g(x)$ and $D^{-j_{0}} T_{-\tilde{x}_{0}} h(x)$ satisfy the corresponding conditions for $i=i_{0}-j_{0}$, $j=0$, and $x_{0}=0$. Thus, assuming Lemma 2.7 holds for $j=0$ and $x_{0}$,

$$
\left|\left(D^{-j_{0}} g * D^{-j_{0}} T_{-\tilde{x}_{0}} h\right)(x)\right| \leq C|\operatorname{det} A|^{-\left(i_{0}-j_{0}\right)(1 / 2+\delta)}\left(1+\rho_{A}(x)\right)^{-R} .
$$

This will show the general case of Lemma 2.7 since

$$
\left(D^{-j_{0}} g * D^{-j_{0}} T_{-\tilde{x}_{0}} h\right)(x)=\left(g * T_{-\tilde{x}_{0}} h\right)\left(A^{-j_{0}} x\right)=(g * h)\left(A^{-j_{0}} x+\tilde{x}_{0}\right) .
$$

Thus, assume that $j=0$ and $x_{0}=0$, take any $x \in \mathbb{R}^{n}$ and decompose $\mathbb{R}^{n}$ into 3 domains

$$
\begin{aligned}
& D_{1}=\left\{y \in \mathbb{R}^{n}: \rho_{A}(y-x)<1\right\}, \\
& D_{2}=\left\{y \in \mathbb{R}^{n}: \rho_{A}(y-x) \geq 1 \text { and } \rho_{A}(y) \leq \rho_{A}(x) /(2 H)\right\}, \\
& D_{3}=\left\{y \in \mathbb{R}^{n}: \rho_{A}(y-x) \geq 1 \text { and } \rho_{A}(y)>\rho_{A}(x) /(2 H)\right\},
\end{aligned}
$$

where $H$ is the constant of the quasi-subadditivity inequality. 
By the vanishing moment condition (2.15) we have

$$
|(g * h)(x)| \leq \int_{\mathbb{R}^{n}}|g(y)-g(x)||h(x-y)| d y \equiv \int_{D_{1}}+\int_{D_{2}}+\int_{D_{3}} .
$$

By (2.13), (2.14), the change of variables, and Proposition 1.4,

$$
\begin{aligned}
\int_{D_{1}} & \leq|\operatorname{det} A|^{i / 2}\left(1+\rho_{A}(x)\right)^{-R} \int_{D_{1}} \rho_{A}(x-y)^{\theta}\left(1+\rho_{A}\left(A^{i}(x-y)\right)\right)^{-R} d y \\
& \leq|\operatorname{det} A|^{i / 2}\left(1+\rho_{A}(x)\right)^{-R} \int_{\mathbb{R}^{n}} \rho_{A}(y)^{\theta}\left(1+\rho_{A}\left(A^{i} y\right)\right)^{-R} d y \\
& \leq|\operatorname{det} A|^{i / 2}\left(1+\rho_{A}(x)\right)^{-R}|\operatorname{det} A|^{-i(1+\theta)} \int_{\mathbb{R}^{n}} \rho_{A}(y)^{\theta}\left(1+\rho_{A}(y)\right)^{-R} d y \\
& \leq C|\operatorname{det} A|^{-i(1 / 2+\theta)}\left(1+\rho_{A}(x)\right)^{-R} .
\end{aligned}
$$

For $y \in D_{2}$, we have

$$
\rho_{A}(x-y) \geq \rho_{A}(x) / H-\rho_{A}(y) \geq \rho_{A}(x) / H-\rho_{A}(x) /(2 H)=\rho_{A}(x) /(2 H),
$$

and subsequently

$$
\left(1+\rho_{A}\left(A^{i}(x-y)\right) \geq|\operatorname{det} A|^{i} \rho_{A}(x-y) \geq|\operatorname{det} A|^{i}\left(1+\rho_{A}(x)\right) /(4 H) .\right.
$$

Hence, by (2.12), (2.14), $\rho_{A}(y) \leq \rho_{A}(x) /(2 H)$ and Proposition 1.4

$$
\begin{aligned}
\int_{D_{2}} & \leq \int_{D_{2}}\left[\left(1+\rho_{A}(x)\right)^{-R}+\left(1+\rho_{A}(y)\right)^{-R}\right] \frac{|\operatorname{det} A|^{i / 2}}{\left(1+\rho_{A}\left(A^{i}(x-y)\right)\right)^{R}} d y \\
& \leq C \frac{|\operatorname{det} A|^{-i(R-1 / 2)}}{\left(1+\rho_{A}(x)\right)^{R}} \int_{\mathbb{R}^{n}} \frac{1}{\left(1+\rho_{A}(y)\right)^{R}} d y \leq C \frac{|\operatorname{det} A|^{-i(R-1 / 2)}}{\left(1+\rho_{A}(x)\right)^{R}} \\
& \leq C|\operatorname{det} A|^{-i(1 / 2+\theta)}\left(1+\rho_{A}(x)\right)^{-R}
\end{aligned}
$$

since $R-1>\theta$.

For $y \in D_{3}$, we have $\rho_{A}(y)>\rho_{A}(x) /(2 H)$ and, hence,

$$
\begin{aligned}
\int_{D_{3}} & \leq \int_{D_{3}}\left[\left(1+\rho_{A}(x)\right)^{-R}+\left(1+\rho_{A}(y)\right)^{-R}\right] \frac{|\operatorname{det} A|^{i / 2}}{\left(1+\rho_{A}\left(A^{i}(x-y)\right)\right)^{R}} d y \\
& \leq C \frac{|\operatorname{det} A|^{i / 2}}{\left(1+\rho_{A}(x)\right)^{R}} \int_{\rho_{A}(x-y) \geq 1} \frac{1}{\rho_{A}\left(A^{i}(x-y)\right)^{R}} d y \\
& =C \frac{|\operatorname{det} A|^{-i(R-1 / 2)}}{\left(1+\rho_{A}(x)\right)^{R}} \int_{\rho_{A}(z) \geq 1} \rho_{A}(z)^{-R} d z \leq C|\operatorname{det} A|^{-i(1 / 2+\theta)}\left(1+\rho_{A}(x)\right)^{-R} .
\end{aligned}
$$

Combining the above estimates yields (2.16) and completes the proof of Lemma 2.7. 
Proof of Lemma 2.5 We consider 2 cases.

Suppose that $|Q| \leq|P|$. Let $i, j \in \mathbb{Z}$ be such that $|Q|=|\operatorname{det} A|^{-i} \leq|\operatorname{det} A|^{-j}=$ $|P|$. Then it is not hard to check that $g(x)=\Psi_{P}\left(x_{P}-x\right)$ and $h(x)=\overline{\Phi_{Q}(x)}$ satisfy the hypotheses of Lemma 2.7 with $R=M, 0<\theta<\min (M-1, \delta)$, and $x_{0}=x_{Q}$. Therefore,

$$
\begin{aligned}
\left|\left\langle\Psi_{P}, \Phi_{Q}\right\rangle\right| & =\left|(g * h)\left(x_{P}\right)\right| \leq C|\operatorname{det} A|^{-(i-j)(1 / 2+\theta)}\left(1+|\operatorname{det} A|^{j} \rho_{A}\left(x_{Q}-x_{P}\right)\right)^{-M} \\
& \leq C|\operatorname{det} A|^{-(i-j)(1 / 2+\theta)}\left(1+|\operatorname{det} A|^{j} \rho_{A}\left(x_{Q}-x_{P}\right)\right)^{-1-\theta} .
\end{aligned}
$$

Suppose that $|Q|>|P|$. Let $i, j \in \mathbb{Z}$ be such that $|Q|=|\operatorname{det} A|^{-j}>|\operatorname{det} A|^{-i}=$ $|P|$. Again, it is not hard to check that hypotheses of Lemma 2.7 with $R=M, 0<$ $\theta<\min (M-1, \delta), x_{0}=x_{P}, g(x)=\overline{\Phi_{Q}\left(x_{Q}-x\right)}$, and $h(x)=\Psi_{P}(x)$, are satisfied. Therefore,

$$
\begin{aligned}
\left|\left\langle\Psi_{P}, \Phi_{Q}\right\rangle\right| & =\left|(g * h)\left(x_{Q}\right)\right| \leq C|\operatorname{det} A|^{-(i-j)(1 / 2+\theta)}\left(1+|\operatorname{det} A|^{j} \rho_{A}\left(x_{Q}-x_{P}\right)\right)^{-M} \\
& \leq C|\operatorname{det} A|^{-(i-j)(1 / 2+\theta)}\left(1+|\operatorname{det} A|^{j} \rho_{A}\left(x_{Q}-x_{P}\right)\right)^{-1-\theta} .
\end{aligned}
$$

Therefore, both cases yield

$$
\begin{aligned}
\left|\left\langle\Psi_{P}, \Phi_{Q}\right\rangle\right| & \leq C\left(1+\frac{\rho_{A}\left(x_{Q}-x_{P}\right)}{\max (|P|,|Q|)}\right)^{-1-\theta} \cdot \begin{cases}(|Q| /|P|)^{1 / 2+\theta} & \text { if }|Q| \leq|P| \\
(|P| /|Q|)^{1 / 2+\theta} & \text { if }|Q|>|P|\end{cases} \\
& =C \kappa_{Q P}(\theta)
\end{aligned}
$$

which completes the proof of Lemma 2.5.

Theorem 2.8 Suppose $\left\{\Psi_{Q}\right\}_{Q} \subset L^{2}\left(\mathbb{R}^{n}\right)$ is affine localized and

$$
\inf \left\{\left\|\Psi_{Q}\right\|_{L^{2}}: Q \in \mathcal{Q}\right\}>0
$$

Then there exists a finite partition of the collections of dilated cubes

$$
\mathcal{Q}=\mathcal{Q}_{1} \cup \cdots \cup \mathcal{Q}_{L}
$$

such that each of the systems $\left\{\Psi_{Q}\right\}_{Q \in Q_{i}}, i=1, \ldots, L$ is a Riesz sequence in $L^{2}\left(\mathbb{R}^{n}\right)$. Furthermore, it is possible to choose this partition to be of the form

$$
\mathcal{Q}_{i}=\left\{A^{-j}\left([0,1]^{n}+k\right):(j, k) \in\left(r_{i}+J \mathbb{Z}\right) \times\left(s_{i}+N \mathbb{Z}^{n}\right)\right\}, \quad i=1, \ldots, L,
$$

where $J, N \in \mathbb{N}, L=J N^{n}$, and $\left(r_{1}, s_{1}\right), \ldots,\left(r_{L}, s_{L}\right)$ are representatives of distinct cosets of $\mathbb{Z} /(L \mathbb{Z}) \times \mathbb{Z}^{n} /\left(N \mathbb{Z}^{n}\right)$. 
Before we give the proof, we wish to emphasize that Theorem 2.8 goes well beyond the original idea of Gröchenig's diagonal dominance approach in [13].

Indeed, we recall that a countable set $\left\{\varphi_{i}: i \in I\right\}$ in a Hilbert space $\mathcal{H}$ is a Riesz sequence if and only if the Gramian matrix $G$ given by its entries $G_{i, j}=\left\langle\varphi_{i}, \varphi_{j}\right\rangle$ defines a bounded and invertible operator on $\ell^{2}(I)$. Furthermore, a sufficient condition for a matrix $\left(a_{i, j}\right)_{i, j \in I}$ defining a bounded positive definite operator on $\ell^{2}(I)$ to be invertible is that $\left(a_{i, j}\right)_{i, j \in I}$ is diagonally dominant, i.e.,

$$
\inf _{i \in I}\left(\left|a_{i, i}\right|-\sum_{j \neq i}\left|a_{i, j}\right|\right)>0 .
$$

The idea of Gröchenig is to decompose an index set $I$ into a finite union of subsets $I=I_{1} \cup \cdots \cup I_{r}$, such that the corresponding Gramian matrix $G$ is decomposed into diagonally dominant, and thus invertible on $\ell^{2}\left(I_{l}\right)$, "sub-Gramian" matrices $G^{(l)}=$ $\left(G_{i, j}\right)_{i, j \in I_{l}}$, where $l=1, \ldots, r$.

This approach does not work in the case of Theorem 2.8, since in Definition 2.1 the decay exponent across the scales is only $1 / 2+\theta$ and one can observe that $1+\theta$ is required for diagonal dominance approach to work. Namely, one can show that for small values $0<\theta<1 / 2$, the matrix $\left(\kappa_{Q P}\right)_{Q, P \in Q}$ does not satisfy Schur's test of boundedness. Therefore, a more delicate approach in showing Theorem 2.8 must be used.

Proof of Theorem 2.8 Let $G=\left(a_{Q P}\right)_{Q, P \in Q}$ be the Gramian matrix given by $a_{Q P}=$ $\left\langle\Psi_{Q}, \Psi_{P}\right\rangle$. By Lemma 2.5, we know that

$$
\left|a_{Q P}\right| \leq C \kappa_{Q P}(\theta) \text { for all } Q, P \in \mathcal{Q} .
$$

Take any $J, N \in \mathbb{N}$ and for a fixed $i=1, \ldots, L=J N^{n}$ consider the collection of cubes $Q^{\prime}=Q_{i}$ given by (2.18). Namely, for $r=0, \ldots, J-1$ and $s \in\{0, \ldots, N-1\}^{n}$ consider

$$
Q^{\prime}=\left\{A^{-j}\left([0,1]^{n}+k\right):(j, k) \in(r+J \mathbb{Z}) \times\left(s+N \mathbb{Z}^{n}\right)\right\} .
$$

Let $\mathcal{A}$ be the operator on $\ell^{2}\left(Q^{\prime}\right)$ associated with the matrix $\left(a_{Q P}\right)_{Q, P \in Q^{\prime}}$. We decompose $\mathcal{A}=\mathcal{A}_{0}+\mathcal{A}_{1}+\mathcal{A}_{2}$ as

$$
\left(\mathcal{A}_{0} s\right)_{Q}=a_{Q Q} s_{Q}, \quad\left(\mathcal{A}_{1} s\right)_{Q}=\sum_{\substack{P \in Q^{\prime} \\|P| \geq|Q| \\ P \neq Q}} a_{Q P} s_{P}, \quad\left(\mathcal{A}_{2} s\right)_{Q}=\sum_{\substack{P \in Q^{\prime} \\|P|<|Q|}} a_{Q P} s_{P},
$$

where $s=\left(s_{P}\right) \in \ell^{2}\left(Q^{\prime}\right)$.

It is clear that $\mathcal{A}_{0}$ is an invertible diagonal operator on $\ell^{2}\left(Q^{\prime}\right)$, since

$$
\inf \left\{a_{Q Q}: Q \in \mathcal{Q}^{\prime}\right\} \geq \inf \left\{\left\|\Psi_{Q}\right\|_{L^{2}}^{2}: Q \in \mathcal{Q}\right\}>0 .
$$

Our goal is to show that for appropriate choice of $J, N \in \mathbb{N}, \mathcal{A}_{1}$ and $\mathcal{A}_{2}$ have very small operator norms on $\ell^{2}\left(Q_{i}\right)$ for every $i=1, \ldots, J N^{n}$. Therefore, $\mathcal{A}$ must be also invertible on $\ell^{2}\left(Q_{i}\right)$ as a small perturbation of an invertible operator $\mathcal{A}_{0}$. 
Indeed, take any $\varepsilon>0$ and let $s=\left(s_{Q}\right) \in \mathcal{Q}^{\prime}$. By the Cauchy-Schwarz inequality

$$
\begin{aligned}
& \sum_{Q \in Q^{\prime}}\left|\left(\mathcal{A}_{1} s\right)_{Q}\right|^{2} \\
& \leq C \sum_{Q \in Q^{\prime}}\left|\sum_{\substack{P \in Q^{\prime} \\
|P| \geq|Q| \\
P \neq Q}}\left(1+\rho_{A}\left(x_{P}-x_{Q}\right) /|P|\right)^{-1-\theta}\left(\frac{|Q|}{|P|}\right)^{1 / 2+\theta}\right| s_{P}||^{2} \\
& \leq C \sum_{Q \in Q^{\prime}}\left[\sum_{\substack{P \in Q^{\prime} \\
|P| \geq|Q| \\
P \neq Q}}\left(1+\rho_{A}\left(x_{P}-x_{Q}\right) /|P|\right)^{-1-\theta}\left(\frac{|Q|}{|P|}\right)^{\theta}\right] \\
& \times\left[\sum_{\substack{P \in Q^{\prime} \\
|P| \geq|Q| \\
P \neq Q}}\left(1+\rho_{A}\left(x_{P}-x_{Q}\right) /|P|\right)^{-1-\theta}\left(\frac{|Q|}{|P|}\right)^{1+\theta}\left|s_{P}\right|^{2}\right]
\end{aligned}
$$

Note that the expression in the first bracket is finite, since

$$
\sum_{\substack{P \in \mathcal{Q} \\|P|=|\operatorname{det} A|^{-j}}}\left(1+\rho_{A}\left(x_{P}-x_{Q}\right) /|P|\right)^{-1-\theta} \leq C<\infty
$$

independent of $j \in \mathbb{Z}$. Therefore, by (2.19) and Lemma 2.6,

$$
\begin{aligned}
& \sum_{Q \in \mathcal{Q}^{\prime}}\left|\left(\mathcal{A}_{1} s\right)_{Q}\right|^{2} \leq C \sum_{P \in \mathcal{Q}^{\prime}}\left|s_{P}\right|^{2} I_{P}, \quad \text { where } \\
& I_{P}=\sum_{\substack{Q \in \mathcal{Q}^{\prime} \\
|Q| \leq|P| \\
Q \neq P}}\left(1+\rho_{A}\left(x_{P}-x_{Q}\right) /|P|\right)^{-1-\theta}\left(\frac{|Q|}{|P|}\right)^{1+\theta} .
\end{aligned}
$$

To estimate $I_{P}$, we pick any $P=A^{-r-J j_{0}}\left([0,1]^{n}+s+N k_{0}\right) \in \mathcal{Q}^{\prime}$, where $j_{0} \in \mathbb{Z}$, $k_{0} \in \mathbb{Z}^{n}$. Next, we split the summation over $|Q|=|P|$ and $|Q|<|P|$ :

$$
\begin{aligned}
& \sum_{\substack{Q \in Q^{\prime} \\
|Q|=|P| \\
Q \neq P}}\left(1+\rho_{A}\left(x_{P}-x_{Q}\right) /|P|\right)^{-1-\theta}\left(\frac{|Q|}{|P|}\right)^{1+\theta} \\
& \quad=\sum_{\substack{k \in \mathbb{Z}^{n} \\
k \neq k_{0}}}\left(1+\rho_{A}\left(N k_{0}-N k\right)\right)^{-1-\theta}=\sum_{k \in \mathbb{Z}^{n} \backslash\{0\}}\left(1+\rho_{A}(N k)\right)^{-1-\theta}<\varepsilon
\end{aligned}
$$


for sufficiently large $N$. Likewise, by Lemma 2.6

$$
\begin{aligned}
\sum_{\substack{Q \in Q^{\prime} \\
|Q|<|P|}}\left(1+\rho_{A}\left(x_{P}-x_{Q}\right) /|P|\right)^{-1-\theta}\left(\frac{|Q|}{|P|}\right)^{1+\theta} \\
=\sum_{j=j_{0}+1}^{\infty} \sum_{k \in Z^{n}} \frac{|\operatorname{det} A|^{J\left(j_{0}-j\right)(1+\theta)}}{\left(1+|\operatorname{det} A|^{r+J j_{0}} \rho_{A}\left(A^{-r-J j}(s+N k)-x_{P}\right)\right)^{1+\theta}} \\
\leq \sum_{j=j_{0}+1}^{\infty} \sum_{k \in Z^{n}} \frac{|\operatorname{det} A|^{J\left(j_{0}-j\right)(1+\theta)}}{\left(1+\rho_{A}\left(A^{J\left(j_{0}-j\right)}(s+k)-A^{r+J j_{0}} x_{P}\right)\right)^{1+\theta}} \\
\leq C \sum_{j=j_{0}+1}^{\infty}|\operatorname{det} A|^{J\left(j_{0}-j\right) \theta}=C|\operatorname{det} A|^{-J \theta} /\left(1-|\operatorname{det} A|^{-J \theta}\right)<\varepsilon
\end{aligned}
$$

for sufficiently large $J$. Combining the above estimates (which are independent of $\left.P \in Q^{\prime}\right)$ with (2.20) yields

$$
\left\|\mathcal{A}_{1} s\right\|_{\ell^{2}\left(\mathcal{Q}^{\prime}\right)}^{2} \leq 2 \varepsilon C\|s\|_{\ell^{2}\left(\mathcal{Q}^{\prime}\right)}^{2} .
$$

Analogously, starting from the estimate

$$
\begin{aligned}
\sum_{Q \in Q^{\prime}}\left|\left(\mathcal{A}_{2} s\right)_{Q}\right|^{2} \leq C \sum_{Q \in \mathcal{Q}^{\prime}} & {\left[\sum_{\substack{P \in \mathcal{Q}^{\prime} \\
|P|<|Q|}}\left(1+\rho_{A}\left(x_{P}-x_{Q}\right) /|Q|\right)^{-1-\theta}\left(\frac{|Q|}{|P|}\right)^{1+\theta}\right] } \\
\times & {\left[\sum_{\substack{P \in \mathcal{Q}^{\prime} \\
|P|<|Q|}}\left(1+\rho_{A}\left(x_{P}-x_{Q}\right) /|Q|\right)^{-1-\theta}\left(\frac{|Q|}{|P|}\right)^{\theta}\left|s_{P}\right|^{2}\right], }
\end{aligned}
$$

and using similar arguments as above one can obtain

$$
\left\|\mathcal{A}_{2} s\right\|_{\ell^{2}\left(Q^{\prime}\right)}^{2} \leq 2 \varepsilon C\|s\|_{\ell^{2}\left(Q^{\prime}\right)}^{2} .
$$

Since $\varepsilon>0$ is arbitrary, this shows that $\mathcal{A}_{1}$ and $\mathcal{A}_{2}$ have small operator norms and consequently $\mathcal{A}$ is invertible on $\ell^{2}\left(Q^{\prime}\right)$ for sufficiently large $J, N \in \mathbb{N}$. This completes the proof of Theorem 2.8 .

Corollary 2.9 follows immediately from Theorem 2.8 and Remark 2.4.

Corollary 2.9 Suppose $\psi(x)$ is a non-zero function such that the $\left\{\psi_{Q}: Q \in Q\right\}$ is affine localized. In particular, it suffices to assume that $\psi(x)$ satisfies

$$
\begin{gathered}
|\psi(x)-\psi(y)| \leq C|x-y|^{\delta}(1+|x|)^{-M / \zeta_{-}} \quad \text { for }|x-y| \leq 1, \\
|\psi(x)| \leq C(1+|x|)^{-M / \zeta_{-}}, \quad \int_{\mathbb{R}^{n}} \psi(x) d x=0,
\end{gathered}
$$


for some $M>1, \delta>0$, and $C>0$.

Then $\left\{\psi_{Q}: Q \in Q\right\}$ is a finite union of pairwise disjoint affine systems, each of which is a Riesz sequence. More precisely, there exist $J, N \in \mathbb{N}$ such that the affine systems

$$
\left\{D^{j} T_{k}\left(D^{r_{i}} T_{s_{i}} \psi\right): j \in J \mathbb{Z}, k \in A^{-r_{i}} N \mathbb{Z}^{n}\right\}, \quad i=1, \ldots, J N^{n}
$$

form a partition of $\left\{\psi_{Q}: Q \in \mathcal{Q}\right\}$ and each system in (2.21) is a Riesz sequence, where $\left(r_{i}, s_{i}\right)$ 's are the same as in Theorem 2.8 .

We remark that in Corollary 2.9 it is not assumed that $\psi(x)$ is a framelet. Nonetheless, the case when $\psi(x)$ is a framelet is probably the most interesting, since it provides a partial answer to the Feichtinger conjecture. In particular, it shows that if a frame wavelet generates affine system $\left\{\psi_{Q}: Q \in \mathcal{Q}\right\}$ localized in the sense of Definition 2.1, then it can be split into a finite union of systems each of which is a Riesz sequence. Since each of these systems is again an affine system (with respect to some power of dilation $A$ and lattice given by (2.21)), a stronger variant of the Feichtinger conjecture holds under the localization assumption.

We also wish to emphasize that Corollary 2.9 is optimal, at least in the qualitative sense. That is, if we remove the hypothesis of $\left\{\psi_{Q}: Q \in \mathcal{Q}\right\}$ being affine localized, then the conclusion of Corollary 2.9 is in general invalid even if $\psi(x)$ is a framelet. Therefore, we will show that the stronger variant of the Feichtinger conjecture for wavelet frames, asserting that affine frame systems can be split into a finite disjoint union of affine Riesz sequences, does not hold without a localization assumption on $\psi(x)$.

Theorem 2.10 There exists a tight frame wavelet $\psi(x) \in L^{2}(\mathbb{R})$ such that the stronger variant of the Feichtinger conjecture fails. More precisely, $\left\{D^{J j} T_{N k} \psi: j, k \in \mathbb{Z}\right\}$ is never a Riesz sequence for any choice of $J, N \in \mathbb{N}$. Moreover, $\psi(x)$ can be chosen to be a semi-orthogonal frame wavelet.

Proof Assume momentarily that we find a nowhere dense set $W \subset[-1 / 2,1 / 2]$ such that $\left\{2^{j} W: j \in \mathbb{Z}\right\}$ partitions $\mathbb{R}$ (modulo null sets) and the sets $\{W+k: k \in \mathbb{Z}\}$ are pairwise disjoint (modulo null sets). Later, we will show how to construct such $W$.

Let $\psi \in L^{2}(\mathbb{R})$ be given by $\hat{\psi}=\chi_{W}$. A standard support argument shows that $D^{j} T_{k} \psi \perp D^{j^{\prime}} T_{k^{\prime}} \psi$ for all $j \neq j^{\prime} \in \mathbb{Z}$ and $k, k^{\prime} \in \mathbb{Z}$. Moreover, since

$$
\sum_{k \in \mathbb{Z}}|\hat{\psi}(\xi+k)|^{2}=\chi_{\tilde{W}}(\xi), \quad \text { where } \tilde{W}=\bigcup_{k \in \mathbb{Z}}(k+W)
$$

$\left\{T_{k} \psi: k \in \mathbb{Z}\right\}$ is a tight frame with constant 1 for its closed linear span, which consists of all $f \in L^{2}\left(\mathbb{R}^{n}\right)$ with supp $\hat{f} \subset W$. Hence, $\left\{D^{j} T_{k} \psi: k \in \mathbb{Z}\right\}$ has the same property for any $j \in \mathbb{Z}$. Since $\left\{2^{j} W: j \in \mathbb{Z}\right\}$ partitions $\mathbb{R}, \psi$ is a semi-orthogonal tight frame wavelet. Moreover, since $W \subset[-1 / 2,1 / 2]$ is nowhere dense, we have that $\sum_{k \in \mathbb{Z}}|\hat{\psi}(\xi+k / N)|^{2}$ must vanish on a set of positive measure and hence $\left\{T_{k N} \psi\right.$ : $k \in \mathbb{Z}\}$ is not a Riesz sequence for any $N \in \mathbb{N}$. Consequently, $\left\{D^{J j} T_{N k} \psi: j, k \in \mathbb{Z}\right\}$ is never a Riesz sequence for any choice of $J, N \in \mathbb{N}$. 
Hence, it remains to show the existence of a set $W$. Let $\left\{W_{k}: k=1,2, \cdots\right\} \subset$ $[-1,-1 / 2] \cup[1 / 2,1]$ be a family of closed nowhere dense sets such that

$$
\bigcup_{k=1}^{\infty} W_{k}=[-1,-1 / 2] \cup[1 / 2,1] \text { and } \sum_{k=1}^{\infty}\left|W_{k}\right|=1 .
$$

Clearly, the $W_{k}$ 's must be pairwise disjoint modulo null sets. Define

$$
W=\bigcup_{k=1}^{\infty} 2^{-k} W_{k} .
$$

An easy verification shows that $W$ has all required properties.

\section{Gabor Frames}

As mentioned in Section 2, Gröchenig in [13] introduced a notion of intrinsically localized frames that are well suited for systems (such as Gabor systems) which are generated by families of unitary operators with compatible actions on $L^{2}\left(\mathbb{R}^{n}\right)$. In particular, he was able to show that if $g$ is in a certain modulation space, then the (possibly irregular) Gabor system $\left\{M_{k} T_{l} g:(k, l) \in X\right\}$ can be written as the finite union of Riesz basic sequences, provided $X$ is the finite union of separated sets. Here, $M_{k} f(x)=e^{2 \pi i\langle x, k\rangle} f(x)$. In particular, the Feichtinger conjecture holds for such Gabor systems.

An earlier result in this direction was provided by Ron and Shen [21], which we describe now. Let $K, L \subset \mathbb{R}^{n}$ be full rank lattices and $K^{*}, L^{*} \subset \mathbb{R}^{n}$ be their dual lattices. The Gabor system $\left\{M_{k} T_{l} g: k \in K, l \in L\right\}$ is said to be sup-adjoint if $K^{*} \subset L$. Ron and Shen showed [21, Theorem 3.5] that if $\left\{M_{k} T_{l} g: k \in K, l \in L\right\}$ is sup-adjoint and a frame for $L^{2}\left(\mathbb{R}^{n}\right)$, then it can be written as the finite union of Riesz basic sequences. Note that in the one-dimensional case, this corresponds to $\left\{M_{k a} T_{l b} g: k, l \in \mathbb{Z}\right\}$ with $a b=1 / N$ for integer $N \geq 1$.

Sticking to regular systems, Casazza et al. [6, Theorem 5.1] were able to generalize the one-dimensional version of Ron and Shen's theorem by showing that if the Gabor system $\left\{M_{k a} T_{l b} g: k, l \in \mathbb{Z}\right\}$ is a frame for $L^{2}(\mathbb{R})$ and $a b$ is rational, then the Gabor system can be written as the finite union of Riesz basic sequences.

Ron and Shen proposed [21] a class of Gabor systems that are higher dimensional analogs of rational Gabor systems, which we describe here.

Definition 3.1 ([21]) Let $K, L$ be full rank lattices in $\mathbb{R}^{n}$. The Gabor system $\left\{M_{k} T_{l} g\right.$ : $k \in K, l \in L\}$ is said to be compressible if $L^{*} \cap K$ has rank $n$.

Remark 3.2 In the one-dimensional case $\left\{M_{k a} T_{l b g}: k, l \in \mathbb{Z}\right\}$ is a compressible Gabor system if and only if $a b$ is rational.

The main result of this short section is the following, which is a generalization of both [21, Theorem 3.5] and [6, Theorem 5.1]. 
Theorem 3.3 Let $K, L \subset \mathbb{R}^{n}$ be full-rank lattices, and $g \in L^{2}\left(\mathbb{R}^{n}\right)$. If $\left\{M_{k} T_{l} g: k \in\right.$ $K, l \in L\}$ is a compressible Gabor system which is a frame for $L^{2}\left(\mathbb{R}^{n}\right)$, then it can be written as the finite union of Riesz basic sequences.

Before proving this theorem, we will develop some tools. The following simple proposition is the key to our development.

Proposition 3.4 Let $K, L \subset \mathbb{R}^{n}$ be full-rank lattices such that $K^{*} \cap L$ has rank $n$ as well. Then there exist $I<\infty$ and $\left\{a_{i}: i=1, \ldots, I\right\}$ such that $\Gamma:=\bigcup_{i=1}^{I}\left(L+a_{i}\right)$ is a lattice, and $K^{*} \cup L \subset \Gamma$.

Proof Choose $\left\{a_{i}: i=1, \ldots, I\right\} \subset K^{*}$ to be a complete set of representatives of $K^{*} /\left(K^{*} \cap L\right)$. Then, $K^{*}=\bigcup_{i=1}^{I}\left(\left(K^{*} \cap L\right)+a_{i}\right)$. We claim $\Gamma=\bigcup_{i=1}^{I}\left(L+a_{i}\right)$ satisfies the conclusion of the theorem. Indeed, $K^{*} \subset \Gamma$ follows immediately from the definition of $\Gamma$, and $L \subset \Gamma$ follows from the fact that one $a_{i}$ is in $L$.

To show $\Gamma$ is a lattice, it suffices to show that $\Gamma$ is a group, since it is clearly a discrete subset of $\mathbb{R}^{n}$. Let $x_{1}=l_{1}+a_{1}$ and $x_{2}=l_{2}+a_{2}$ be in $\Gamma$, where $a_{1}, a_{2}$ are intended to represent arbitrary elements of $\left\{a_{i}: i=1, \ldots, I\right\}$, and $l_{j}$ are arbitrary elements of $L$. Then since $K^{*} /\left(K^{*} \cap L\right)$ is a group, there is a number $k \in\{1, \ldots, I\}$ such that $\left(K^{*} \cap L+a_{1}\right)-\left(K^{*} \cap L+a_{2}\right)=\left(K^{*} \cap L+a_{k}\right)$, i.e., $a_{k}-\left(a_{1}-a_{2}\right)=l \in K^{*} \cap L$. Then, $x_{1}-x_{2}=l_{1}-l_{2}-l+a_{k} \in L+a_{k} \subset \Gamma$.

Remark 3.5 In the one-dimensional case, if $\left\{M_{k} T_{l} g: k \in \mathbb{Z}, l \in(p / q) \mathbb{Z}\right\}$ with $0<p / q<1$ is a compressible Gabor system, then Proposition 3.4 yields $\Gamma=$ $\bigcup_{i=0}^{p-1}(p / q \mathbb{Z}+i)$. In their proof that one-dimensional compressible Gabor systems satisfy the Feichtinger conjecture, Casazza et al. also found this oversampling, but wrote it as $\Gamma=\bigcup_{i=0}^{p-1}(p / q \mathbb{Z}+i / q)$.

We now show that the condition on the lattices in Proposition 3.4 is equivalent to the Gabor system being compressible.

Proposition 3.6 Let $K, L \subset \mathbb{R}^{n}$ be full-rank lattices. Then $K^{*} \cap L$ is rank $n$ if and only if $K \cap L^{*}$ is rank $n$.

Proof Suppose $K^{*} \cap L$ has rank $n$. Obtain $\Gamma$ from Proposition 3.4. Since $L \cup K^{*} \subset \Gamma$, it follows that $\Gamma^{*} \subset L^{*} \cap K$. Hence, since $\Gamma^{*}$ has rank $n$, so does $L^{*} \cap K$.

We now prove the main result in this section.

Proof of Theorem 3.3 Let $\Gamma$ be as in Proposition 3.4. Then, $\left\{M_{k} T_{\gamma} g: k \in K, \gamma \in\right.$ $\Gamma\}$ is a sup-adjoint Gabor system. Since $L \subset \Gamma$, a lower frame bound is satisfied, and since the cardinality of $I$ is finite, and $\Gamma=\bigcup_{i \in I}\left(L+a_{i}\right)$, an upper frame bound is also satisfied. By [21, Theorem 3.5], $\left\{M_{k} T_{\gamma} g: k \in K, \gamma \in \Gamma\right\}$ can be written as the finite union of Riesz basic sequences. Hence, since $\left\{M_{k} T_{l} g: k \in K, l \in L\right\} \subset\left\{M_{k} T_{\gamma} g\right.$ : $k \in K, \gamma \in \Gamma\}$, the result holds. 


\section{Frames of Translates}

In this section we investigate the following question. Suppose that

$$
\mathcal{S}_{f}^{\Gamma}:=\{f(x+k): k \in \Gamma\}, \quad \text { where } \Gamma \subset \mathbb{R}^{n}
$$

is a frame for its closed linear span. Can $\mathcal{S}_{f}^{\Gamma}$ be written as the finite union of Riesz basic sequences? There is a large collection of results that can be applied to this question. One purpose of this section is to show what types of results can be proven using known techniques, and to show where the limits of known techniques seem to be. Of particular interest is Theorem 4.16, which provides evidence against the Feichtinger conjecture, even in this restricted setting.

We begin by applying the results of Gröchenig [13] to this setting. Let $X$ be a separated subset of $\mathbb{R}^{n}$, i.e., $\inf _{x, y \in X, x \neq y}|x-y|=\delta>0$. Recall that a collection of vectors $\left\{e_{x}: x \in X\right\} \subset \mathcal{H}$ is said to be intrinsically localized if for some $s>n$,

$$
\left|\left\langle e_{x}, e_{y}\right\rangle\right| \leq C(1+|x-y|)^{-s}
$$

for all $x, y \in X$.

Proposition 4.1 Let $f \in L^{2}\left(\mathbb{R}^{n}\right)$ be such that $|f(x)| \leq(1+|x|)^{-s}$ for some $s>n$. If $\Gamma$ is a separated subset of $\mathbb{R}^{n}$, then $\mathcal{S}_{f}^{\Gamma}$ is intrinsically localized. Consequently, if $\mathcal{S}_{f}^{\Gamma}$ is a frame for its closed span, then it can be written as the finite union of Riesz basic sequences.

Proof It suffices to show for $k, l \in \Gamma$ that

$$
|\langle f(x+k), f(x+l)\rangle|=|\langle f(x), f(x+(l-k))\rangle| \leq C(1+|l-k|)^{-s} .
$$

Write $m=l-k$. Let $I_{1}=\left\{x \in \mathbb{R}^{n}:|x|<|x+m|\right\}$, and $I_{2}=\mathbb{R}^{n} \backslash I_{1}$. Then for $x \in I_{1},|x+m| \geq \frac{1}{2}|m|$, and for $x \in I_{2},|x| \geq \frac{1}{2}|m|$. Thus,

$$
\begin{aligned}
|\langle f(x), f(x+m)\rangle| & =\left|\int_{I_{1}} f(x) \overline{f(x+m)} d x+\int_{I_{2}} f(x) \overline{f(x+m)} d x\right| \\
& \leq \int_{I_{1}}|f(x)||f(x+m)| d x+\int_{I_{2}}|f(x)||f(x+m)| d x \\
& \leq(1+|m / 2|)^{-s}\left(2 \int|f(x)| d x\right)
\end{aligned}
$$

as desired.

For the second statement, since $\mathcal{S}_{f}^{\Gamma}$ is a frame, the number of points of $\Gamma$ inside each cube of side length 1 is uniformly bounded. In fact, it is enough for $\mathcal{S}_{f}^{\Gamma}$ to be a Bessel sequence, see Lemma 4.7. Hence, $\Gamma=\bigcup_{i=1}^{M} \Gamma_{i}$, with each $\Gamma_{i}$ separated. Therefore, one can apply [13, Theorem 2$]$ to each $\Gamma_{i}$ separately and the result follows. 
Remark 4.2 By analyzing the proof of [13, Theorem 2] more carefully (see also Theorem 2.8 above), in the case that $\Gamma$ is a lattice and $\mathcal{S}_{f}^{\Gamma}$ is intrinsically localized, one can show that the Riesz basic sequences can all be chosen to be translates of a function by a lattice. Thus, the stronger version of the Feichtinger conjecture holds in this case. We omit the details.

In the case that $\Gamma$ is a lattice, one can weaken the hypotheses of Proposition 4.1 somewhat. Denote

$$
\sigma_{f}^{\Gamma}(\xi)=\sum_{\gamma \in \Gamma^{*}}|\hat{f}(\xi+\gamma)|^{2},
$$

where we will suppress the dependence on $\Gamma$ when it is obvious from context. We begin by recalling the following; see for example [5, Theorem 2.2].

Theorem 4.3 Let $f \in L^{2}\left(\mathbb{R}^{n}\right)$, and $\Gamma \subset \mathbb{R}^{n}$ be a full-rank lattice. Then,

(i) $\mathcal{S}_{f}^{\Gamma}$ is a frame for its closed linear span if and only if there exists $0<A \leq B<$ $\infty$ such that $A \chi_{U} \leq \sigma_{f}^{\Gamma} \leq B \chi_{U}$ almost everywhere, where $U=\left\{\xi \in \mathbb{R}^{n}\right.$ : $\left.\sigma_{f}^{\Gamma}(\xi)>0\right\}$.

(ii) $\mathcal{S}_{f}^{\Gamma}$ is a Riesz basic sequence if and only if there exists $0<A \leq B<\infty$ such that $A \leq \sigma_{f}^{\Gamma} \leq B$ almost everywhere.

An immediate application of Theorem 4.3 yields

Proposition 4.4 Let $f \in L^{2}\left(\mathbb{R}^{n}\right), \Gamma \subset \mathbb{R}^{n}$ be a full-rank lattice. If $\mathcal{S}_{f}^{\Gamma}$ is a frame for its closed linear span and there exists $\eta=\left(\eta_{1}, \ldots, \eta_{n}\right) \in[0,1]^{n}, \epsilon>0$ such that

$$
E:=\eta+B(0, \epsilon) \subset \operatorname{supp}\left(\sigma_{f}^{\Gamma}\right),
$$

then $\mathcal{S}_{f}^{\Gamma}$ can be written as the finite union of Riesz basic sequences.

Proof Choose a lattice $\Lambda$ of rank $n$ satisfying $\Gamma^{*} \subset \Lambda$ and

$$
\sum_{\lambda \in \Lambda} \chi_{E}(\xi+\lambda) \geq 1, \text { a.e. }
$$

Then, $A \leq \sigma_{f}^{\Lambda} \leq B$ almost everywhere, so $\delta_{f}^{\Lambda^{*}}$ is a Riesz basic sequence. Since $\Gamma=\bigcup_{i \in I}\left(\Lambda^{*}+a_{i}\right)$, and $\mathcal{S}_{f}^{\Lambda^{*}+a_{i}}$ are all Riesz basic sequences, the proposition follows.

Remark 4.5 Proposition 4.4 applies, in particular, if $\hat{f}=\chi_{[a, b]}$. In this case,

$$
\left|\left\langle f, T^{k} f\right\rangle\right|=\frac{1}{2 \pi|k|}\left|e^{2 \pi i k b}-e^{2 \pi i k a}\right|,
$$

so for almost every choice of $a, b, f$ is not intrinsically localized. However, for every choice of $a$ and $b, \mathcal{S}_{f}^{Z}$ is a frame and can be written as the finite union of Riesz basic sequences. 
With a little more work, we can improve on Propositions 4.4 and 4.1 in the onedimensional case. Recall the following theorem of Montgomery and Vaughan [19, Theorem 1, Chapter 7], [20].

Theorem 4.6 Suppose that $\lambda_{1}, \ldots, \lambda_{N}$ are distinct real numbers, and suppose that $\delta>0$ is chosen so that $\left|\lambda_{n}-\lambda_{m}\right| \geq \delta$ whenever $n \neq m$. Then for any coefficients $a_{1}, \ldots, a_{N}$, and any $T>0$,

$$
(T-1 / \delta) \sum_{n=1}^{N}\left|a_{n}\right|^{2} \leq \int_{0}^{T}\left|\sum_{n=1}^{N} a_{n} e^{2 \pi i \lambda_{n} t}\right|^{2} d t \leq(T+1 / \delta) \sum_{n=1}^{N}\left|a_{n}\right|^{2} .
$$

We will also need the following lemma, which has several variants in the literature, e.g., [16, Lemma 1]. We include a proof for completeness.

Lemma 4.7 Let $f \in L^{2}(\mathbb{R})$ and $\Lambda=\left\{\lambda_{n}: n \in \mathbb{Z}\right\}$. If there exists a constant $B$ such that for every $m \in \mathbb{Z}$

$$
\sum_{n \in \mathbb{Z}}\left|\left\langle e^{2 \pi i \lambda_{n} t} f, e^{2 \pi i \lambda_{m} t} f\right\rangle\right|^{2} \leq B
$$

then the number of points $\lambda_{n}$ inside each interval of length 1 is uniformly bounded.

Proof We prove the contrapositive. By standard arguments, there exists an $\epsilon>0$ such that for all $\eta$ with $|\eta| \leq \epsilon$,

$$
\left.\left.\left|\int_{\mathbb{R}} e^{2 \pi i \eta t}\right| f(t)\right|^{2} d t\right|^{2} \geq \frac{1}{2}\|f\|^{2} .
$$

Suppose that the number of $\lambda_{n}$ inside an interval of length one is not bounded. Then the number of $\lambda_{n}$ inside intervals of length $\epsilon$ is also not bounded. Choose $\mu_{k}$ such that the number of $\lambda_{n}$ inside $A_{k}:=\left[\mu_{k}-\epsilon / 2, \mu_{k}+\epsilon / 2\right]$ is at least $k$. Choose $\lambda_{n_{k}} \in A_{k} \cap \Lambda$ arbitrarily. Then, by (4.5) it follows that

$$
\sum_{n \in \mathbb{Z}}\left|\left\langle e^{2 \pi i \lambda_{n_{k}} t} f, e^{2 \pi i \lambda_{n} t} f\right\rangle\right|^{2} \geq \frac{k}{2}\|f\|^{2} .
$$

Hence, $\Lambda$ does not satisfy the hypotheses of the lemma.

Theorem 4.8 Let $f \in L^{2}(\mathbb{R})$ be such that there exists $a>0$ and an interval I such that $a \chi_{I} \leq|\hat{f}|$, and let $\Gamma$ be an arbitrary subset of $\mathbb{R}$. If $S_{f}^{\Gamma}$ is a frame for its closed span, then $S_{f}^{\Gamma}$ can be written as the finite union of Riesz basic sequences.

Proof By Parseval's identity, $\left\langle f\left(x+\gamma_{1}\right), f\left(x+\gamma_{2}\right)\right\rangle=\left\langle e^{2 \pi i \gamma_{1} \xi} \hat{f}(\xi), e^{2 \pi i \gamma_{2} \xi} \hat{f}(\xi)\right\rangle$. Thus, since $S_{f}^{\Gamma}$ is a frame for its closed span, the hypotheses of Lemma 4.7 are satisfied, and $\Gamma$ can be written as the finite union of separated sequences. In particular, one can write $\Gamma$ as the finite union of sets $\Gamma=\Gamma_{1} \cup \cdots \cup \Gamma_{N}$ such that for each $1 \leq i \leq N$, $\delta_{i}:=\inf \left\{|\gamma-\lambda|: \gamma, \lambda \in \Gamma_{i}, \gamma \neq \lambda\right\}$ satisfies $\frac{1}{\delta_{i}}<|I|$. Clearly, the interval $[0, \bar{T}]$ in Theorem 4.6 can be replaced by any interval of length $T$, so $\left\{f(x+\gamma): \gamma \in \Gamma_{i}\right\}$ is a Riesz basic sequence with lower bound $a^{2}\left(|I|-\frac{1}{\delta_{i}}\right)$. 
Corollary 4.9 If $\left\{e^{2 \pi i \lambda \xi}: \lambda \in \Lambda\right\}$ is a Fourier frame, i.e., a frame for $L^{2}([0,1])$, then it can be written as the finite union of Riesz basic sequences.

Corollary 4.9 was also observed by Christensen and Lindner. See the discussion after Theorem 2.7 of their paper [8].

\subsection{Frames of Integer Translates}

For the rest of this section we will restrict our attention to the special case of frames of translates $\delta_{f}^{\Gamma}$ with $\Gamma=\mathbb{Z}$. In this setting, we can show that the following relationship holds between frames of translates and pavings of Laurent operators.

Proposition 4.10 Let $f \in L^{2}(\mathbb{R})$ be such that $\mathcal{S}_{f}^{\mathbb{Z}}$ is a frame for its closed span and $\hat{f}$ is a function supported on $[0,1]$. Let $\varphi$ be the 1 -periodic extension of $|\hat{f}|^{2}$ on $[0,1]$. If the Laurent operator $L_{\varphi}$ satisfies the paving conjecture, then $\mathcal{S}_{f}^{\mathbb{Z}}$ can be written as the finite union of Riesz basic sequences.

Proof By Proposition 4.3, $\hat{f}$ is bounded, so $\varphi \in L^{\infty}(\mathbb{T})$, where $\mathbb{T}=\mathbb{R} / \mathbb{Z}$. Hence, $\varphi$ defines a Laurent operator $L_{\varphi}: L^{2}(\Pi) \rightarrow L^{2}(\Pi)$ given by $L_{\varphi}(f)=f \varphi$.

According to [6, Proposition 3.1] and its proof, a frame $\left\{f_{k}\right\}_{k \in \mathbb{Z}}$ can be written as the finite union of Riesz basic sequences if the operator $S: \ell^{2}(\mathbb{Z}) \rightarrow \ell^{2}(\mathbb{Z})$, whose matrix is given by

$$
\left\langle S e_{k}, e_{j}\right\rangle= \begin{cases}\left\langle f_{k}, f_{j}\right\rangle & k \neq j, \\ 0 & k=j,\end{cases}
$$

can be paved, where $\left\{e_{k}\right\}_{k \in \mathbb{Z}}$ is the standard orthonormal basis of $\ell^{2}(\mathbb{Z})$. Applying this to the frame $\left\{e^{2 \pi i k \xi} \hat{f}(\xi): k \in \mathbb{Z}\right\}$ yields $S$ of the form

$$
\left\langle S e_{k}, e_{j}\right\rangle= \begin{cases}\left.\widehat{\left(|\hat{f}|^{2}\right.}\right)(k-j)=\hat{\varphi}(k-j) & k \neq j \\ 0 & k=j .\end{cases}
$$

This is exactly the matrix of $L_{\varphi}$ minus its diagonal, see [2]. Hence, the result follows.

Theorem 4.8 should be contrasted with the following elementary observation.

Example 4.11 Let $U$ be a nowhere dense subset of $[0,1]$ with positive measure, $\hat{f}=\chi_{U}$. Then for any natural number $N>0, \bigcup_{k \in \mathbb{Z}}(U-k / N)$ is nowhere dense since the finite union of nowhere dense sets is nowhere dense and $U$ is bounded. Consequently,

$$
\sum_{k \in \mathbb{Z}} \chi_{U}(\xi+k / N)=0
$$

on a set of positive measure. Hence, by Theorem $4.3, \mathcal{S}_{f}^{N Z}$ is not a Riesz basic sequence. 
While there is a characterization of when $\{f(x+k): k \in \mathcal{K}\}$ is a Riesz basic sequence for $\mathcal{K} \subset \mathbb{Z}[5]$, it is still difficult to decide whether $\{f(x+k): k \in \mathcal{K}\}$ is a Riesz basic sequence when $\Gamma$ is an irregular subset of $\mathbb{Z}$ and $f$ is poorly localized. In particular, for $f$ as generally defined as in Example 4.11, the authors do not know whether the Feichtinger conjecture is satisfied. We formalize the most natural question along this line of thought as:

Question 4.12 Suppose $\hat{f}=\chi_{U}$, and $\{f(x+k): k \in \mathbb{Z}\}$ is a frame for its closed linear span. Can $\{f(x+k): k \in \mathbb{Z}\}$ be written as the finite union of Riesz basic sequences?

Of course, by Proposition 4.10, a positive solution to the paving problem for Laurent operators would imply a positive answer to Question 4.12.

We now present a specially chosen $f$ of the form $\hat{f}=\chi_{U}$ with the property that any set $\mathcal{K} \subset \mathbb{Z}$ for which $\{f(x+k): k \in \mathcal{K}\}$ is a Riesz basic sequence must be "quite strange". While this example does not answer Question 4.12, it will suggest a line of attack for studying it. We will need to recall some machinery in order to define $\hat{f}=\chi_{U}$. We follow the development given in [14].

Let $u=\left\{u_{n}: n=1,2, \ldots\right\}$ be a sequence of points in the torus $\mathbb{T}=\mathbb{R} / \mathbb{Z}$. The sequence $u$ is said to be uniformly distributed if

$$
\lim _{N \rightarrow \infty} \frac{\#\left\{1 \leq n \leq N: u_{n} \in I\right\}}{N}=|I|
$$

for every arc $I$ in the torus. For a finite, nonempty set $S \subset \mathbb{T}$ (possibly with multiplicity), we define the discrepancy of $S$ to be

$$
\operatorname{Discr}(S)=\sup _{I \subset \mathbb{T}}\left|\frac{\#(S \cap I)}{\# S}-\right| I|| .
$$

The $N$-term discrepancy of the sequence $u$ is defined as

$$
D_{N}(u)=\operatorname{Discr}\left(\left\{u_{n}: 1 \leq n \leq N\right\}\right)
$$

We will need two results.

Theorem 4.13 ([19, Koksma's Inequality $]) \quad$ For any sequence of points $u_{1}, \ldots, u_{N}$ in $\mathbb{T}$, and any function $f: \mathbb{T} \rightarrow \mathbb{R}$ of bounded variation,

$$
\left|\frac{1}{N} \sum_{n=1}^{N} f\left(u_{n}\right)-\int_{0}^{1} f(t) d t\right| \leq \operatorname{Var}(f) \operatorname{Discr}\left(\left\{u_{n}: 1 \leq n \leq N\right\}\right),
$$

where $\operatorname{Var}(f)$ is the total variation of $f$.

Theorem 4.14 ([17]) For any $\epsilon>0$, the $N$-term discrepancy of $u_{n}=n \alpha \bmod 1$ satisfies

$$
D_{N}(u) \leq C_{\alpha} N^{-1} \log ^{2+\epsilon} N
$$

for almost all $\alpha$, where $C_{\alpha}$ depends only on $\alpha$. 
Definition 4.15 Let $g: \mathbb{N} \rightarrow[0, \infty)$. We say that $\mathcal{K} \subset \mathbb{Z}$ satisfies the $g(N)$ arithmetic progression condition if for every $\delta>0$ there exist $M \in \mathbb{Z}$ and $N, \ell \in \mathbb{N}$ such that

(i) $\ell<\delta g(N)$, and

(ii) $\{M, M+\ell, \ldots, M+N \ell\} \subset \mathcal{K}$.

Theorem 4.16 There exists a set $U \subset[0,1]$ such that if $\mathcal{K} \subset \mathbb{Z}$ satisfies the $g(N)=$ $N^{1 / 2} \log ^{-3} N$ arithmetic progression condition, then $\{f(x+k): k \in \mathcal{K}\}$ is not a Riesz basic sequence, where $\hat{f}=\chi_{U}$.

Proof Let $u=u_{N}(\xi)=\{\xi, 2 \xi, \ldots, N \xi\}$. By Theorem 4.14 with $\epsilon=1$, for almost all $\xi$ and all $N$,

$$
D_{N}(u) \leq \frac{C(\xi)}{N} \log ^{3} N
$$

Choose $K$ such that

$$
0<|\{\xi \in[0,1]: C(\xi) \leq K\}|<1,
$$

and let $U=\{\xi \in[0,1]: C(\xi) \leq K\}$. (This choice of $K$ is possible since $C$ is not a constant function. However, it formally does not matter for this proof, since if $C$ were constant, then one could choose any subset of $[0,1]$.)

Let $\delta>0$ be arbitrary and find $M, N$ and $\ell$ such that $\ell N^{-1 / 2} \log ^{3} N<\delta$ and such that $\{M, M+\ell, \ldots, M+\ell N\} \subset \mathcal{K}$. Let $h(\xi)=e^{2 \pi i \ell \xi}$. By Theorem 4.13,

$$
\left|\frac{1}{N} \sum_{k=1}^{N} h(k \xi)-0\right| \leq \operatorname{Var}(h) \operatorname{Discr}\left(u_{N}(\xi)\right) .
$$

Therefore,

$$
\left|\frac{1}{N} e^{2 \pi i M \xi} \sum_{k=1}^{N} h(k \xi)\right| \leq 2 \pi K \ell \frac{1}{N} \log ^{3} N .
$$

Multiplying both sides by $\sqrt{N}$ and integrating yields

$$
\left(\int_{U}\left|\frac{1}{\sqrt{N}} e^{2 \pi i M \xi} \sum_{k=1}^{N} h(k \xi)\right|^{2}\right)^{1 / 2} \leq 2 \pi|U|^{1 / 2} K \ell N^{-1 / 2} \log ^{3} N<2 \pi K \delta .
$$

So, since $\sum_{k=1}^{N}\left(\frac{1}{\sqrt{N}}\right)^{2}=1$ and $\delta>0$ is arbitrary, it follows from the definition of Riesz basis that $\{f(x+k): k \in \mathcal{K}\}$ is not a Riesz basic sequence.

Remark 4.17 Theorem 4.16 should be compared to [15, Theorem 5.4 (b)], where a similar negative result is shown for pavings of Laurent operators with certain symbols. Since the paving conjecture for Laurent operators implies the Feichtinger conjecture for frames of translations (Proposition 4.10), Theorem 4.16 is a stronger negative example. The examples given in [15, Theorem 5.4 (b)] were considered natural candidates for being possible counterexamples to the paving conjecture. It was proven in [2] that not all of such operators are counterexamples. 
Remark 4.18 If one randomly assigns each integer to one of $L$ subsets $\mathcal{K}_{1}, \ldots \mathcal{K}_{L}$, then with probability one, for each $i$ and $L$ there will exist $M_{i}$ such that

$$
\left\{M_{i}, M_{i}+1, \ldots, M_{i}+L\right\} \subset \mathcal{K}_{i} .
$$

Hence, with $f$ as in Theorem 4.16 and $\mathbb{Z}$ partitioned as $\bigcup_{i=1}^{L} \mathcal{K}_{i}$, the probability that the set $\left\{f(x+k): k \in \mathcal{K}_{i}\right\}$ is a Riesz basic sequence is zero.

One should also compare the $g(N)=N^{1 / 2} \log ^{-3} N$ arithmetic progression condition with van der Waerden's theorem, which states that, given a partition of the integers $\left\{\mathcal{K}_{1}, \ldots, \mathcal{K}_{L}\right\}$, there exists an $i$ such that $\mathcal{K}_{i}$ has arbitrarily long arithmetic progressions. One might hope that there is a quantitative version of van der Waerden's theorem that would give the existence of $g(N)=N^{1 / 2} \log ^{-3} N$ arithmetic progressions, but this is not the case. The current best quantitative version is given by Gowers [12], where we use the notation $a \uparrow b$ to denote $a^{b}$, with natural rules for order of operation.

Theorem 4.19 ([12, Theorem 18.2]) Let $0<\gamma \leq 1 / 2$, let $k$ be a positive integer, let $P \geq 2 \uparrow 2 \uparrow \gamma^{-1} \uparrow 2 \uparrow 2 \uparrow(k+9)$, and let $A$ be a subset of $\{1, \ldots, P\}$ of size at least $\gamma P$. Then $A$ contains an arithmetic progression of length $k$.

Corollary 4.20 Suppose $\mathbb{Z}$ is partitioned into $L$ subsets $\mathcal{K}_{1}, \ldots, \mathcal{K}_{L}$. Let $g(N)=$ $2 \uparrow 2 \uparrow L \uparrow 2 \uparrow 2 \uparrow(N+9)$. Then there exists an $i$ such that the set $\mathcal{K}_{i}$ satisfies the $g(N)$ arithmetic progression condition.

Proof With $g$ as above, let $\delta=\frac{1}{N}$. Choose $\gamma=\frac{1}{L}, k=N$ and $P=2 \uparrow 2 \uparrow L \uparrow 2 \uparrow 2 \uparrow$ $(k+9)$ as in Theorem 4.19. By Theorem 4.19, there is an $i$ such that $\mathcal{K}_{i}$ contains an arithmetic progression of length $N$. Now the "jump" in the progression $\ell$ satisfies $\ell \leq P / N$. Therefore, for this arithmetic progression, $\ell \leq \delta g(N)$. Since there are only finitely many $\mathcal{K}_{i}$ 's, there exists a $\mathcal{K}_{i}$ for which the $g(N)$ arithmetic progression condition is satisfied.

We end this section by recalling the following theorem of Bourgain and Tzafriri, which remains the strongest indicator that the answer to Question 4.12 is "yes". Recall that, for a set $K \subset \mathbb{Z}$, the density of $K$ is defined to be

$$
\operatorname{dens}(K)=\lim _{n \rightarrow \infty} \frac{\#(K \cap[1, n])}{n},
$$

provided that the limit exists.

Theorem 4.21 ([1]) Let $U \subset \mathbb{T}$ be a set of positive measure. There exists a set $K \subset \mathbb{Z}$ such that $\operatorname{dens}(K)>0$ and $\left\{e^{2 \pi i k x} \chi_{U}: k \in K\right\}$ is a Riesz basic sequence.

In this section, we have shown that there are techniques available from the interplay of number theory with harmonic analysis that can be used to address the Feichtinger conjecture in the case of frames of translates. It is natural to wonder 
whether more sophisticated tools in this area can be used to settle the Feichtinger conjecture for frames of translates. It would be of particular interest to find the optimal functions $g(N)$ such that Theorem 4.16 and Corollary 4.20 hold. Indeed, if $g(N)$ in Theorem 4.16 could be chosen to be smaller than $g(N)$ in Corollary 4.20 , then the Feichtinger conjecture would be disproven. There is currently a bit of a gap in the available estimates.

Acknowledgment The authors thank the referee for providing the reference [15].

\section{References}

[1] J. Bourgain and L. Tzafriri, Invertibility of "large" submatrices with applications to the geometry of Banach spaces and harmonic analysis. Israel J. Math. 57(1987), 137-224.

[2] $\longrightarrow$ On a problem of Kadison and Singer. J. Reine Angew. Math. 420(1991), 1-43.

[3] M. Bownik, Anisotropic Hardy spaces and wavelets. Mem. Amer. Math. Soc. 164(2003), no. 781

[4] M. Bownik and K.-P. Ho, Atomic and Molecular Decompositions of Anisotropic Triebel-Lizorkin Spaces. Trans. Amer. Math. Soc. (to appear).

[5] P. Casazza, O. Christensen, and N. Kalton, Frames of translates. Collect. Math. 52(2001), 35-54.

[6] P. Casazza, O. Christensen, A. Lindner, and R. Vershynin, Frames and the Feichtinger conjecture. Proc. Amer. Math. Soc. 133(2005), 1025-1033.

[7] P. Casazza and R. Vershynin, Kadison-Singer meets Bourgain-Tzafriri. preprint. http://www.math.missouri.edu/ pete/pdf/kadison-singer.pdf

[8] O. Christensen and A. Lindner, Decomposition of Riesz frames and wavelets into a finite union of linearly independent sets. Linear Algebra Appl. 355(2002), 147-159.

[9] M. Frazier and B. Jawerth, Decomposition of Besov spaces. Indiana Univ. Math. J. 34(1985), 777-799.

[10] $\underset{34-170}{\longrightarrow}$ A discrete transform and decomposition of distribution spaces. J. Funct. Anal. 93(1990), $34-170$.

[11] M. Frazier, B. Jawerth, and G. Weiss, Littlewood-Paley Theory and the Study of Function Spaces. CBMS Regional Conference Ser., \#79, American Math. Society (1991).

[12] W. T. Gowers, A new proof of Szeméredi's theorem. Geom. Funct. Anal. 11(2001), 465-588.

[13] K. Gröchenig, Localized frames are finite unions of Riesz sequences. Adv. Comput. Math. 18(2003), $149-157$.

[14] C. S. Güntürk, Approximating a bandlimited function using very coarsely quantized data: improved error estimates in sigma-delta modulation. J. Amer. Math. Soc. 17(2004), 229-242

[15] H. Halpern, V. Kaftal and G. Weiss, Matrix pavings and Laurent operators. J. Operator Theory 16(1986), 355-374.

[16] S. Jaffard, A density criterion for frames of complex exponentials. Michigan Math. J. 38(1991), 339-348.

[17] L. Kuipers and H. Niederreiter, Uniform Distribution of Sequences. Pure and Applied Mathematics Wiley-Interscience, New York, 1974.

[18] P.-G. Lemarié-Rieusset, Projecteurs invariants, matrices de dilatation, ondelettes et analyses multi-résolutions. Rev. Mat. Iberoamericana 10(1994), 283-347.

[19] H. L. Montgomery, Ten Lectures on the Interface Between Analytic Number Theory and Harmonic Analysis. CBMS Regional Conference Series in Mathematics 84, American Mathematical Society, Providence, RI, 1994.

[20] H. L. Montgomery and R. C. Vaughan, Hilbert's inequality. J. London Math. Soc. (2) 8(1974), 73-82.

[21] A. Ron and Z. Shen, Weyl-Heisenberg frames and Riesz bases in $L_{2}\left(\mathbb{R}^{d}\right)$. Duke Math. J. 89(1997), 237-282.

Department of Mathematics

University of Oregon

Eugene, OR 97403-1222

U.S.A.

e-mail: mbownik@uoregon.edu
Department of Mathematics and Computer Science

Saint Louis University

221 N. Grand Blvd.

St. Louis, MO 63103

U.S.A.

e-mail: speegled@slu.edu 